\title{
Disintegration of the Astroglial Domain Organization May Underlie the Loss of Reality Comprehension in Schizophrenia: A Hypothetical Model
}

\author{
Bernhard J. Mitterauer \\ Volitronics Institute for Basic Research, University of Salzburg, Salzburg, Austria \\ Email: mitterauer.b@gmail.com
}

How to cite this paper: Mitterauer, B.J. (2019) Disintegration of the Astroglial Domain Organization May Underlie the Loss of Reality Comprehension in Schizophrenia: A Hypothetical Model. Open Journal of Medical Psychology, 8, 15-35. https://doi.org/10.4236/ojmp.2019.82002

Received: March 13, 2019

Accepted: April 14, 2019

Published: April 17, 2019

Copyright () 2019 by author(s) and Scientific Research Publishing Inc. This work is licensed under the Creative Commons Attribution International License (CC BY 4.0).

http://creativecommons.org/licenses/by/4.0/ (c) (i) Open Access

\begin{abstract}
A pathophysiological model of the loss of reality comprehension in schizophrenia is proposed. Based on a formalism it is hypothesized that astroglial domains exert an information-categorizing function which becomes progressively lost in the schizophrenic process, caused by functional and structural disintegration of astroglial domains. Unconstrained synaptic neurotransmission functionally disintegrates the astroglial domains. Microdomains located between perisynaptic astroglial processes and the synaptic membrane are interpreted as elementary functional units categorizing synaptic information processing. Unconstrained diffusion of neurotransmitters into the extrasynaptic space leads to the dysfunction of microdomain formation. In parallel, atrophic processes of astroglia progressively break up the connectivity between synaptic and extrasynaptic compartments disrupting astroglial domains. Basically, the organization of astroglia into definite functional and structural units (modules) of astroglial-synaptic information processing may enable the brain to comprehend ontological realms such as subjects and objects in the environment and their distinct qualities. This fundamental capability of cognition is lost in schizophrenia. It is suggested that this ontological confusion of astroglial domain boundaries progressively disorganizes reality comprehension and may represent the basic pathology in schizophrenia underlying the main symptoms of the disorder. Finally, the testing of the model is shortly discussed.
\end{abstract}

\section{Keywords}

Schizophrenia, Astroglial Domains, Disintegration, Loss of Reality Comprehension 


\section{Introduction}

Schizophrenia is a chronic mental disorder characterized by disruption in cognition and emotion affecting language, perception, thought, and the sense of self [1]. The core symptoms are delusions and hallucinations, thought disorder, catatonic symptoms, and affective flattening [2] [3]. Psychotic symptoms such as delusions and hallucinations reflect the confusion about the loss of boundaries between the patient and the external world, since the patient is incapable of distinguishing between his/her own thoughts and perceptions of the environment [4]. Although the pathogenesis of schizophrenia still is unknown, existing models may partly be explanatory. These models include the neurodevelopmental hypothesis, genetic inheritance, environmental factors, impairments in cognitive function, neurodegeneration, and alterations in neurotransmission [1] [5] [6]. The neurodevelopmental hypothesis suggests that structural brain changes in the prenatal or perinatal period predispose the later development of schizophrenia. In addition, significant environmental stress affects early brain development. Severe cognitive impairment is characteristic of schizophrenia. Patients suffering from schizophrenia have problems thinking clearly and initiating speech due to memory problems and dysfunctions in information processing. This cognitive impairment further affects decision making and problem solving. Neurodegeneration and degeneration of glia cells identified in schizophrenic brains may be responsible for cognitive impairment, as suggested by the neurodegenerative model of schizophrenia [7]. Furthermore, alterations in neurotransmission are proposed as the main factor in the etiology of schizophrenia, leading to various neurotransmitter hypotheses. Since all of these components may interact in various intensities during the course of the illness, schizophrenia may have a multifactorial genesis [8].

Most studies have directed their investigations on the mechanism of schizophrenia towards neuronal dysfunctions and impairments and have defined schizophrenia as a "neuro-centric" disorder [9]. However, along with the development of genetics and systematic biology approaches in recent years, the key role of glial cells in the etiopathophysiology of schizophrenia has been demonstrated [10] [11] [12]. The present study intends to further elaborate on my pathophysiological model of schizophrenia developed over the past years [13]-[19]. Here, I will focus on astroglia not referring to oligodendroglia and microglia which also play an important role in the pathophysiology of schizophrenia [10] [19] [20]. Since astroglial cells represent the "linchpin" [21] comprising all glial cells (oligodendrocytes, microglia) in the glial networks, it may be of interest to develop a pathophysiological model of schizophrenia based on astroglia.

The paper is organized as follows: starting out with the hypothesis that the disintegration of the astroglial domain organization may underlie the loss of reality comprehension in schizophrenia, following with the description of the structural and functional organization of astroglial domains and microdomains. Next, a combinatorial formalism of the qualitative categorization of information processing 
corresponding to the operations of astroglial domains is proposed, and the functional disintegration of astroglial domains and microdomains is described based on experimental findings. I suggest that the organization of astroglial domains into definite functional and structural units (modules) may enable the brain to comprehend ontological realities in their distinct qualities in the environment. Exactly this elementary capability of cognition is lost in schizophrenia and may be responsible for the loss of reality comprehension underlying the core symptoms of the disorder. In conclusion, testing the significance of the model is shortly discussed.

\section{Hypothetical Model}

The pathophysiology of schizophrenia underlying the loss of reality comprehension may be caused by the disintegration of the astroglial domain organization. Basically, the domain of a single astrocyte embodies a non-overlapping territory [22] [23] [24] operating as a functional island [25]. Astrocytic peripheral processes (APP) and neurons together build tripartite synapses [26]. Microdomains are mainly located on APP [27] and represent elementary functional units that may not only integrate information [28], but also categorize synaptic information processing determined by the modulatory function of astroglia. Generally, categorization is defined as a process in which ideas and objects are differentiated [29].

Dependent on neuronal activation a hierarchy of astroglial domains from nanodomains, microdomains, cellular domains to superdomains can be generated [30]. This hierarchic organization of astroglial domains may enable the brain to categorize synaptic information in levels of increasing complexity and to distinguish qualitative differences between subjects and objects in the environment, as well as between itself and the environment on the behavioral level. As shown below, the generation of astroglial domains is based on a formalism for categorizing domain qualities in a hierarchical order. I hypothesize that the functional and structural disintegration of astroglial domains leads to the inability to categorize information from the inner and outer environment. Therefore, schizophrenic patients cannot grasp the significance or meaning of information, thus losing the comprehension of reality.

\section{Astroglial Domain Organization}

Cortical and hippocampal astrocytes are organized in non-overlapping spatial domains [22] [23] [24] [25]. It is estimated that a single human astrocyte can cover from about 270,000 to 2 million synapses within its domain [22] [23] [31]. Stem processes emanating from the soma of the astrocyte ramify progressively to finally generate a dense matrix of APP [27] which enwrap neuronal synaptic structures, termed tripartite synapses [26]. Importantly, the synaptic ensheathment by APP increases with neuronal activity and is highly dynamic, since APPs can extend and retract from dendritic spines in a time scale of minutes, leading 
to changes of the degree of synaptic coverage [27] [32] [33] [34].

Significantly, Halassa and colleagues [25] characterized the territory or domain of a single astrocyte as functional islands of synapses in which the signals of neighboring synapses can be coordinated by an individual astrocyte. Rodent and human astrocytes respond to neurotransmitters through rises of intracellular calcium, which is compartmentalized and restricted to individual astrocytes [35] [36] [37] [38]. Dendrites cross the domain of up to hundred different astrocytes integrating various synaptic inputs modulated by independent astroglial cells [39]. Basically, astrocytes act as a complementary neuromodulatory system [40] [41] [42]. The domain organization may enable astrocytes to integrate and filter separate volumes of synaptic activity, providing an analysis and output that reflects the unique complexity of the glial-neuronal network [38] [40].

Astroglial domains function basically autonomously and can be described in a hierarchical order [30]. Whereas nanodomains represent only very small astrocyte-neuron contacts, microdomains enwrap an individual synapse or a group of synapses. A single astrocyte consists of hundreds of independent microdomains, as identified in the Bergmann glial cells in the cerebellum [28]. Microdomains and even nanodomains embody dynamic and plastic structures [43] that may operate as elementary functional units for categorizing synaptic information processing. Cellular domains embody a higher level of the astroglial domain organization by enwrapping larger synaptic aggregates such as glomeruli in the olfactory cortex, ocular dominance columns of the visual cortex or "barrels" in the somatosensory cortex. The functional organization of these astroglial domains is dependent on neuronal activation. Since astrocytes are interconnected via gap junctions, astroglial networks of higher complexity can be formed, termed macrodomains and superdomains. The latter may comprise cortical areas or gyri [30].

Kozachkov and Michmizos [44] proposed an architecture of the astroglial domain organization shown in Figure 1. Astroglial domains are described as circles arranged in a hierarchical order (a). The domain organization of five astrocytes is displayed. The APP of each astrocyte contact neuronal synapses (tripartite synapses, TPS) forming the structure of a domain. Astrocytes are interconnected via gap junctions (GJ) building an astroglial network.

\section{Microdomains}

Grosche and coworkers [26] identified in Bergmann glial cells of the cerebellum close contact sites with the neurons, called "glial microdomains". These subcellular compartments have a complex surface of thin membrane sheets enwrapping only few synapses. Each microdomain is electrotonically independent of the stem process from which it emanates and also from neighboring domains. The authors conclude that the glial microdomain compartmentalizes ensembles of synapses representing a morphological unit that may synchronize local synaptic activity [45]. 


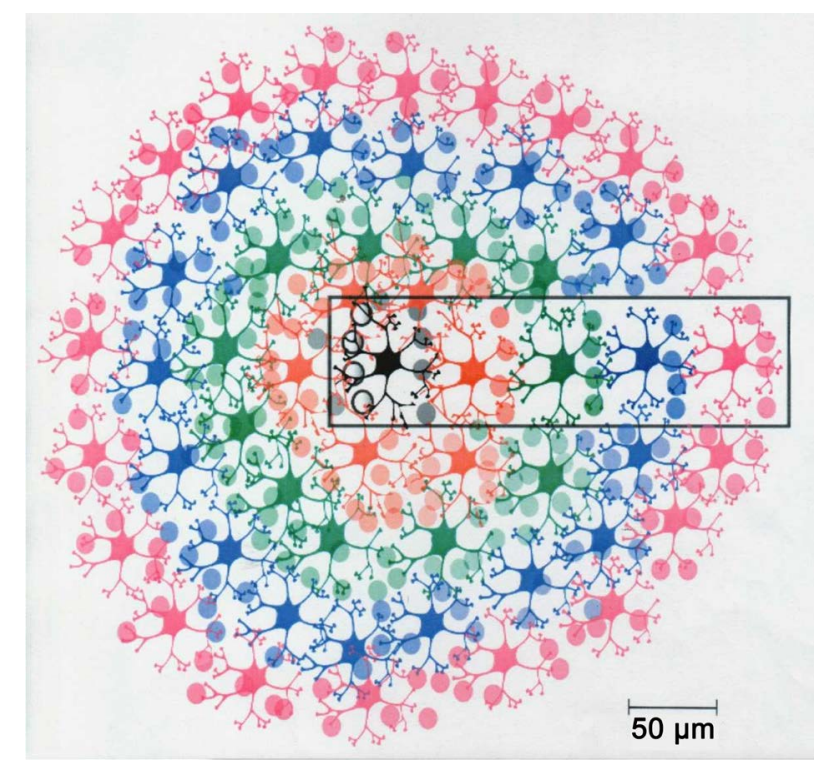

Figure 1. Architecture of the astroglial domain organization.

Basically, APPs contain elements fundamental for astrocyte signaling such as receptors, transmitters, and transporters. In addition, APPs are equipped with mitochondria and glycogen (energy production), actin filaments (motility), and gap junctions (intracellular communication). Microdomains are located along APPs. Panatier and colleagues [46] showed that rapid kinetics of $\mathrm{Ca}^{2+}$ transients in "astrocyte functional compartments" (microdomains) along APPs result from the stimulation of a single synapse. Astrocytes detect small levels of locally released neurotransmitters simultaneously with the postsynaptic neuron. Therefore, like neurons astrocytes process synaptic information at the level of individual synapses. Importantly, perisynaptic microdomains occur particularly extensive at the margin of each astrocyte domain where processes of adjacent domains interdigitate [21].

Figure 2 outlines the formation of two glutamate microdomains [47]. A single synapse is ensheathed by a single astrocyte. Glutamate (GLU) is released from the presynapse activating postsynaptic glutamatergic receptors such as N-methyl-D-asparate receptors (NMDAR). Glutamatergic receptors (NMDAR, mGLUR 2/5) are extrasynaptically activated by GLU released from the pre- and postsynapse, generating glutamate microdomains (MD). Plasma membrane glutamate transporters (EAAT1/2) on the astroglial process bind and transport GLU, inhibiting the spillover of GLU from the synaptic cleft to the extracellular space. In parallel, $\mathrm{K}^{+}$and $\mathrm{Na}^{+}$channels in the astroglial process control molecular homeostasis, forming potassium $\left(\mathrm{K}^{+}\right)$and sodium $\left(\mathrm{Na}^{+}\right)$microdomains. Importantly, a computational model elucidates the mechanisms of cation-domain formation fundamental for homeostatic astroglial-synapse interactions [48].

I propose that the generation of perisynaptic astroglial compartments or microdomains may not only represent "contextual guidance" [49], but may basically categorize synaptic properties as demonstrated in the visual cortex [50]. If 


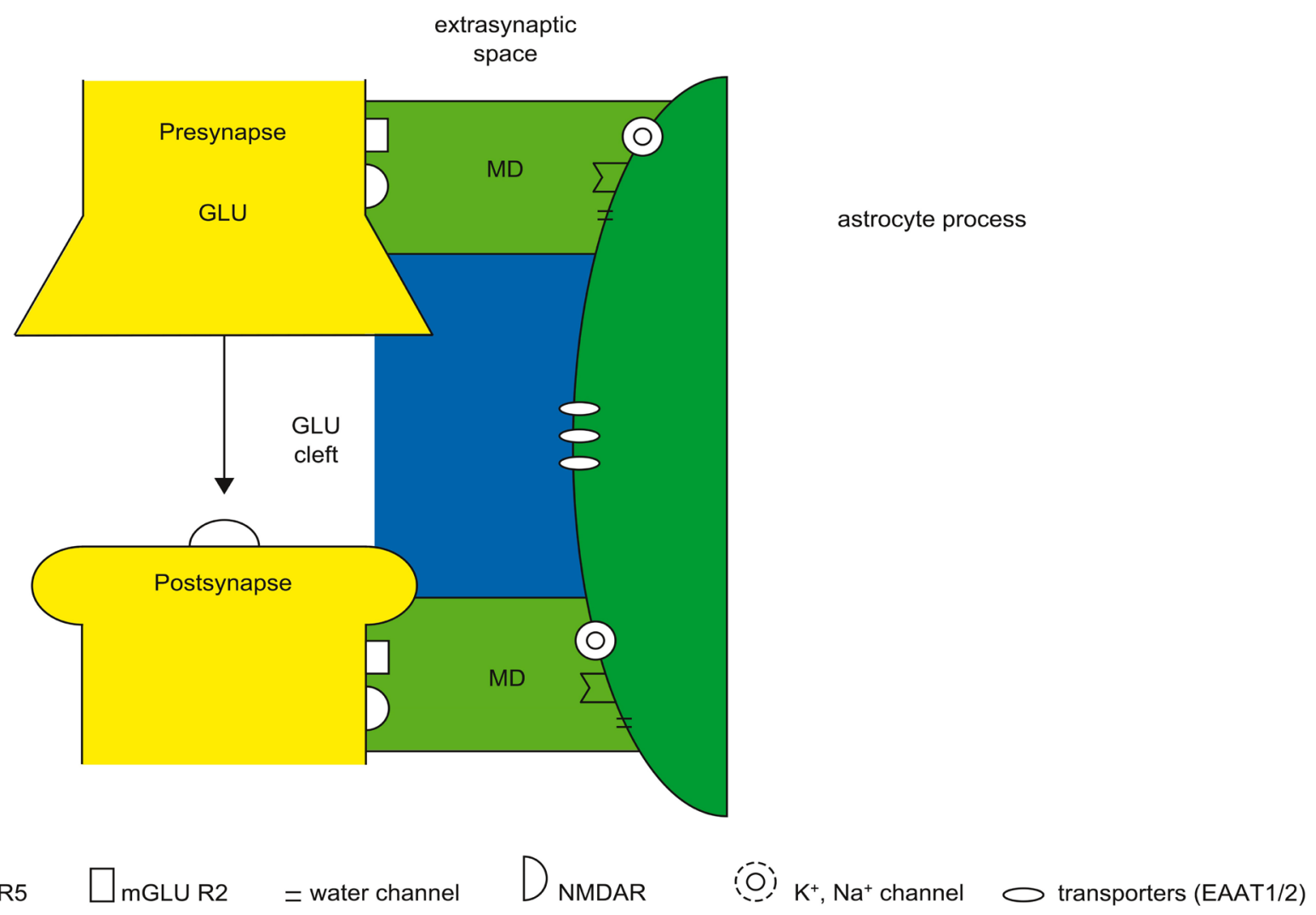

YmGLU R5 $\square$ mGLU R2 = water channel $D_{\text {NMDAR }}$ ioj $\mathrm{K}^{+}, \mathrm{Na}^{+}$channel $\square$ transporters (EAAT1/2)

Figure 2. Formation of glutamate microdomains.

the formation of glial microdomains of various transmitter-modulator systems is disturbed, the boundaries between domains become loosen up leading to the disintegration of astroglial domains.

\section{Neuromodulator Microdomains}

Although neuromodulator microdomains are as yet not experimentally identified, I suggest that these domains may exert elementary operations of qualitative information processing. Since all types of receptors for neurotransmitters are localized in the membrane of perisynaptic astroglial processes [51], microdomains for neuromodulators such as serotonin, dopamine, norepinephrine, and acetylcholine may also be formed. In spite of the fact that it is difficult to define a specific function of each neuromodulator [52], qualitative information processing may represent a common function [21]. For example, the serotonergic system is associated with qualitatively different emotional states experienced as love and hate, or joy and sadness [53]. The key function of dopamine is conferring motivational salience [54]. This means that dopamine "qualifies" the perceived motivational prominence according to its desirability or aversiveness of an outcome and drives the behavior of the organism toward or away from achieving this outcome [55]. In other words, dopamine encodes the precision of alternative action programs and respond to novel environmental events finding out new action programs [54]. 
Furthermore, acetylcholine is associated with memory consolidation, attention, and uncertainty computation [56]. The latter has been demonstrated in computer simulations [52]. Acetylcholine enhances sensory information, decreases recurrent activity and "qualifies" learning and memory exerting a basic role in uncertainty-mediated inference computation [57]. Since acetylcholine coordinates neuronal information processing in various brain areas, it may underlie complex behavior [58]. Importantly, the noradrenergic system mediates the switch between exploration and exploitation behaviors allowing for behavioral adaptation [59], where astrocytes act as a gate for stimulus-specific plasticity [49] [60]. Norepinephrine also exerts effects on memory consolidation and streaming the priority of information in a given behavioral state [61]. Most importantly, norepinephrine may in concert with other neuromodulators be responsible for our awareness of being a distinct person [53].

Fundamentally, since neuromodulators cooperate in generating distinct programs for selecting a specific behavior, their functional integration requires a theoretical basis for our understanding of these systems [62]. Whereas in tripartite synapses neuromodulators such as norepinephrine and acetylcholine modulate synaptic properties and activity, extrasynaptic microdomains may additionally compartmentalize and integrate ensembles of synapses, as demonstrated in Bergmann glial cells in the cerebellum by the Grosche group [27]. Therefore, it is obvious that glial microdomains may be formed for neurotransmitters and neuromodulators in various areas of the brain.

\section{Formal Structure of the Astroglial Domain Organization}

I propose a combinatorial formalism, called morphogrammatics [63]. It categorizes the astroglial domain organization as a tree corresponding to the hierarchical arrangement of co-existing astroglial domains [64] (Figure 3). Starting out with the domain quality a (first level), at the second level two domains with the same ( $a$ a) and different ( $a b)$ qualities are generated. At the third level domains with three different $(a b c)$ qualities are combined. The tree represents the combinatorics of all domains with five qualities generating five levels of complexity (Note, the qualities a ... e define the value ranges 1-2 (a); 3-4 (b); 5-6 (c); 7-8 (d); 9-10 (e)).

The generation rule of domains is as follows: a domain $x$ with $\mathrm{n}+1$ qualities (a ... e) may be generated from domain $y$ on the first $n$ qualities. For example, the domain $\mathrm{a} b \mathrm{a} \mathrm{c}$ (third level of the tree) can be generated from $\mathrm{a} b \mathrm{a}$, but not from a a b. In Figure 3 the generation rule is drawn in lines between the columns. Importantly, the position of each quality in each column is decisive. Given the astroglial domain organization in the visual cortex where astrocytes are organized into functional units [65], where several astrocytes respond to an individual orientation stimulus and two astrocytes may respond to completely different visual orientations [50], the formalism proposed describes and interprets these biological findings. Basically, the modulation and integration of information 


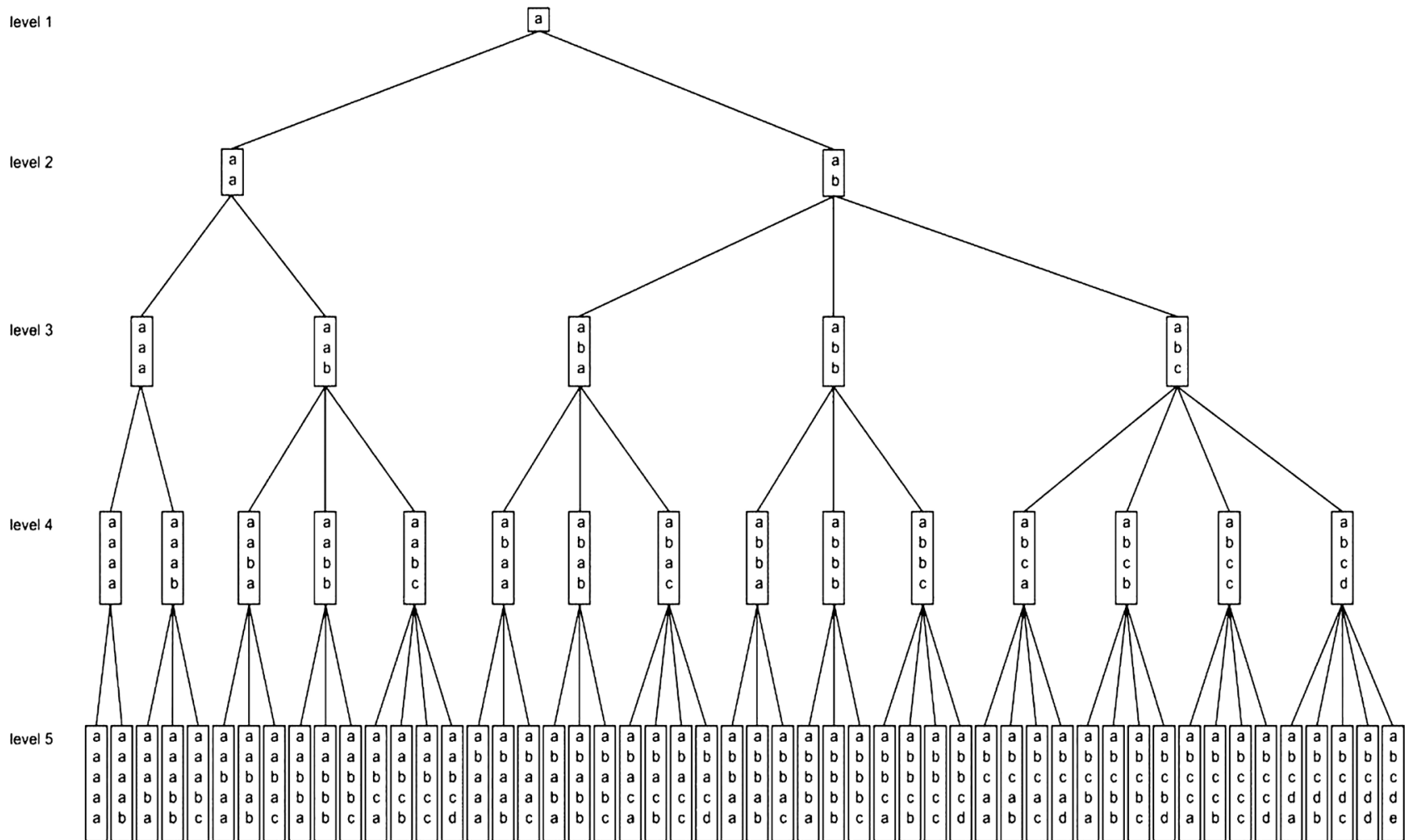

Figure 3. Formal structure of the astroglial domain organization.

processing in astroglial domains may represent a larger layer of complexity of computation in the brain [39] [40] [66]. However, the significance of the astroglial domain organization may be based on qualitative information processing by structuring information into categories [67]. This capability of the brain may be lost in schizophrenia.

\section{Disintegration of the Astroglial Domain Organization in Schizophrenia}

There is convincing evidence for the crucial role of glial cells in the etiopathophysiology of schizophrenia [9] [11] [68] [69]. Here, I will focus on astroglia, the key player in glial-neuronal information processing [21]. I hypothesize that in schizophrenia the astroglial domain organization is disintegrated, caused by dysfunctions and structural impairments of astrocyte-synaptic and -extrasynaptic information processing, leading to the incapability to qualitatively categorize information from the inner and outer world. Therefore, patients with schizophrenia cannot "grasp" the significance of information so that the comprehension of reality is lost, as frequently observed in hallucinations and delusions.

\section{Imbalanced Neurotransmission in Tripartite Synapses}

As described above, perisynaptic astroglial processes express receptors, transporters, ion channels, and various other proteins required for synaptic transmission [31]. The activation of astrocytic receptors elevates $\mathrm{Ca}^{2+}$ concentration leading 
to the production of gliotransmitters such as GLU, GABA (gamma-amino-butyric acid), adenosine/adenosine-tri-phosphate, and D-serine [70]. Clinical investigations found decreased levels of the gliotransmitter D-serine in the plasma of patients with schizophrenia [71] [72]. The decreased expression of D-serine may be determined by mutations associated with schizophrenia [73], and also by changes in the biogenesis and catabolism of D-serine. Basically, proteins fundamental for homeostatic functions are down-regulated in brains with schizophrenia, causing dysbalanced synaptic transmission [74]. In addition, the increased synthesis of chondroitin sulfate proteoglycans, a component of the extracellular matrix identified in the entorhinal cortex and amygdala, may imbalance synaptic transmission [75]. Kynurenic acid is an NMDAR (N-methyl-D-asparate receptor) antagonist like phencyclidine and ketamine and can provoke psychotic symptoms. Significantly, the dopaminergic system is regulated by the kynurenic pathway. The increase in kynurenic acid level potentiates the firing of midbrain dopaminergic neurons and the release of dopamine shown in rodent models [76] [77]. Therefore, kynurenic acid may play a role in synaptic imbalance of the dopaminergic system in schizophrenia.

In transgenic animals a down-regulation of serine racemase, a D-serine synthesizing enzyme, has been found. These animals express the Disrupted-InSchizophrenia 1 (DISC1) gene in astrocytes [73], and alternated interplay with serine racemase caused a depletion of D-serine inciting schizophrenia-like behavior. Importantly, many human mutations possibly responsible for schizophrenia lead to a hypofunction of NMDAR co-agonist binding site that either decreases its affinity or directly impairs D-serine availability [78] [79]. In parallel, the cholinergic system is dysregulated affecting NMDAR activity and NMDARdependent functions [49] [80] [81].

My model of synaptic imbalance in the pathophysiology of schizophrenia focuses on dysfunctional astrocytic transmitter receptors and the disruption of gliotransmitter release from astrocytes. Therefore, astroglia lose their modulatory function in tripartite synapses leading to an unconstrained neurotransmission so that gaps between the neuronal and glial networks arise. The unconstrained neurotransmitter flux may impair the oligodendro-axonic interactions because of toxic effects on axonal hyperactivation by unconstrained neurotransmission [18]. Given that tripartite synapses are located on APPs in the astroglial domain, disrupted astrocyte-synapse information processing disintegrates the astroglial domain organization. In parallel, atrophic processes may structurally disintegrate astroglial domains [7] (discussed below).

Significantly, Mahdavi and coworkers [82] developed an extended mathematical model of an imbalanced tripartite synapse based on my model of the pathophysiology of schizophrenia. It was shown that the lack of glutamatergic feedback from astrocytes caused by non-functional astrocytic receptors and the increased nitric monoxide (NO) from the postsynaptic neuron lead to unconstrained release of GLU by the presynaptic terminal. Excess of GLU and lack of 
D-serine lead to the decreased amplitude and fluctuation of NMDAR current and to lower frequency of excitatory postsynaptic potentials. The authors suggest that NMDARs are dysregulated because of the impaired neurotransmission and through excessive NO-dependent phosphorylation of NMDAR.

\section{Dysfunction of Glial Microdomain Formation}

Figure 4 outlines the loss of glial microdomain formation caused by unconstrained diffusion of GLU in the extrasynaptic space. If the expression of astroglial glutamate transporters (EAAT1/2) is decreased [83] [84], the diffusion of GLU in the extrasynaptic space becomes unconstrained, leading to a dysfunction of microdomain formation [47]. Abnormal protein expression of EAATs was found in the hippocampus, the anterior cingulate cortex, and the prefrontal cortex in brains with schizophrenia. Although many studies found a reduction of EAAT2 levels in schizophrenia, unchanged or increased levels of EAAT2 have been observed as well [85] [86]. Changes of EAAT levels differ in astrocytes and neurons. In astrocytes EAAT1 levels may be decreased, but no so in neurons. Interestingly, animals lacking EAAT1 displayed a schizophrenia-like behavior [87]. Basically, dysfunctions of transporters, receptors, and ion channels located

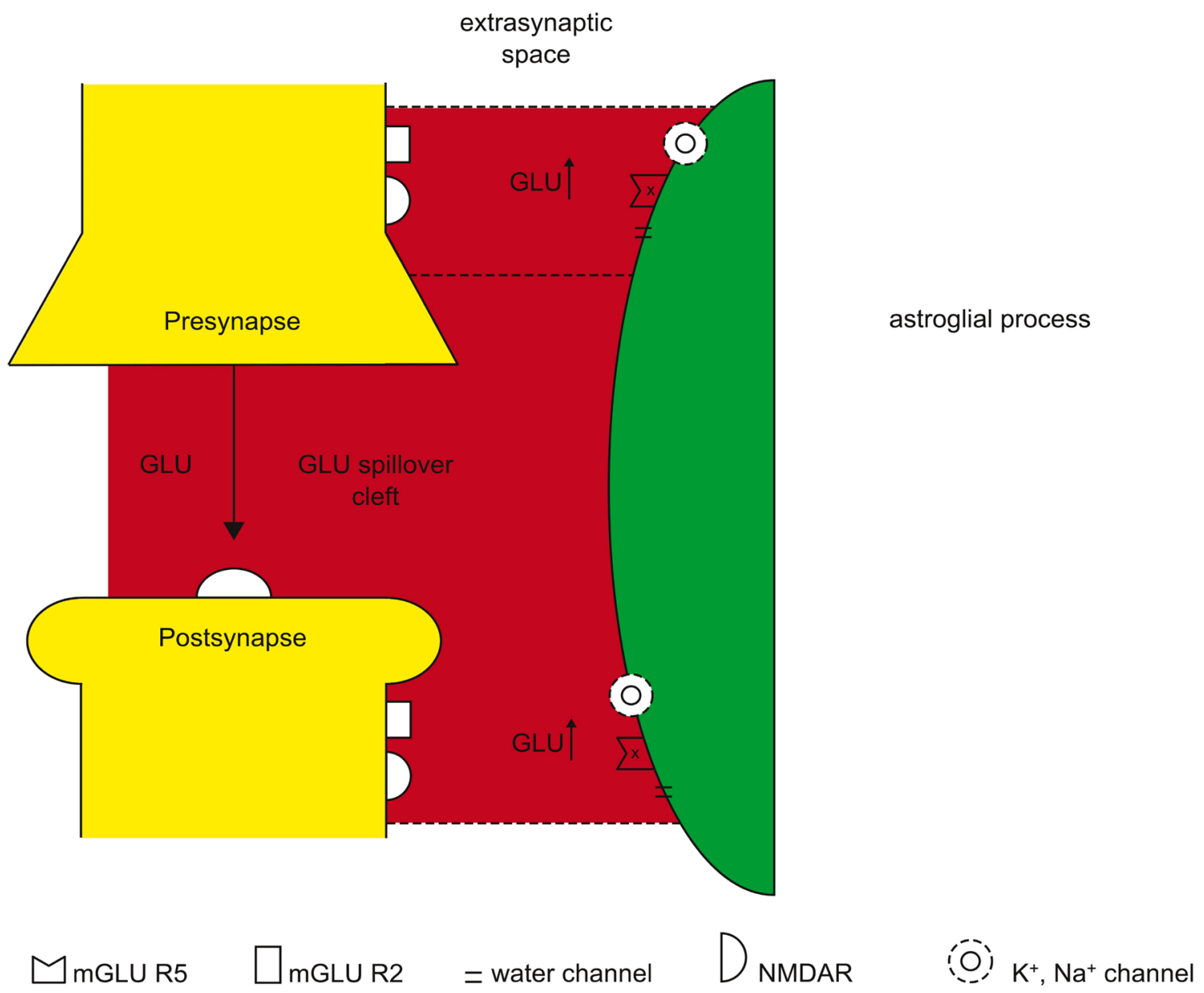

Figure 4. Loss of microdomain formation. 
on the perisynaptic astroglial processes may affect the formation of microdomains.

In addition, disturbance of the glutamate-glutamine cycle in astrocytes has been reported [88]. In a rodent phencyclidine model of schizophrenia the expression of the cystine-glutamate exchanger $(\mathrm{xCT})$ that controls extrasynaptic concentration was upregulated [89]. The enhanced release of GLU in the synaptic cleft, as proposed in my model of synaptic imbalance in schizophrenia [14] [15] [16], spills over in the extrasynaptic space because of changes in astroglial GLU receptors and transporter expression. This mechanism may lead to an unconstrained diffusion of GLU between synapses and microdomains so that microdomains disintegrate and lose their information integrating function.

\section{Structural Impairments of Astroglia}

In parallel to synaptic and extrasynaptic dysregulations outlined above, structural impairments of the astrocyte may progressively disintegrate the domain organization in schizophrenia. A general decrease in numbers of astrocytes and glial fibrillary acid protein (GFAP) expression is documented in numerous studies in human brains with schizophrenia [90]. In various regions of postmortem brain a decrease in the total number of Nissl-stained astrocytes was found [91]. Reduced size of astroglial mitochondria is associated with the duration of the disease and astroglial dysfunction [92]. Increased release of S100 $\beta$ may also indicate astroglial dystrophic changes [7]. Moreover, alterations of astroglial markers and loss of astroglial cells have been identified in brains with schizophrenia causing a decrease of glycogen metabolism, dysregulation of glutamate homeostasis [93], and decreased expression of astroglial EAAT1/2 glutamate transporters, as identified in the prefrontal cortex and hippocampus [94].

Furthermore, alterations of the extracellular matrix were found in schizophrenic brains. Changes of Reelin expression and a substantial increase in the astroglial synthesis of chondroitin sulphate proteoglycans are found in the amygdala, entorhinal cortex and hippocampus, but not in bipolar disorder [95]. These alterations may disintegrate extracellular homeostasis and impair synaptic connectivity [68]. Excitingly, recent experiments on human glial chimeric mice using human glial progenitor cells (hGPCs) demonstrated that the differentiation of astrocytes is delayed and astrocytes show abnormal morphologies [69]. These experiments suggest that a cell-autonomous gliopathology significantly contributes to the genesis and development of juvenile-onset schizophrenia. Impaired astrocytic differentiation from schizophrenia hGPCs may further affect hypomyelination, because of the metabolic dependence of mature oligodendrocytes on astrocytes. Schizophrenia-derived hGPCs suppress glial differentiation-associated genes which encode for synaptic proteins associated with synaptic neurotransmission [96]. Notably, the assessment of astrocytic differentiation revealed in contrast to control astrocytic processes that schizophrenia astrocytic processes left empty spaces. This finding indicates that the astroglial domain structure is disconnected [69]. 

domains

b) uncategorized neuronal net

Figure 5 shows a schema of the loss of astroglial domains caused by astroglial atrophy. Three astrocytes form three astroglial domains interconnected by gap junctions (GJ). Peripheral astroglial processes contact neurons forming tripartite synapses (TPS) (a). Astroglial atrophy leads to an unmodulated neuronal net because of the loss of astroglial cells (b).

\section{Loss of Reality Comprehension in the Schizophrenic Process}

As discussed above, astroglial dysfunctions and impairments disrupt the interaction with the neuronal system losing the modulatory and structuring function of neuronal information processing by astroglia. This leads to an unconstrained neurotransmission and a generalization of information processing in various brain areas [14] [17] [18]. In parallel, the structural impairments destruct the astroglial domains causing progressive cognitive disabilities.

Given that the disintegration of the astroglial domain organization runs in a progressive process in schizophrenia, the symptomatology is determined by the degree of synaptic dysfunction and the structural impairment of astroglial domains. In the prodrome of the illness the patient is still able to recognize the realities in the environment, but significant behavioral changes occur [97]. In

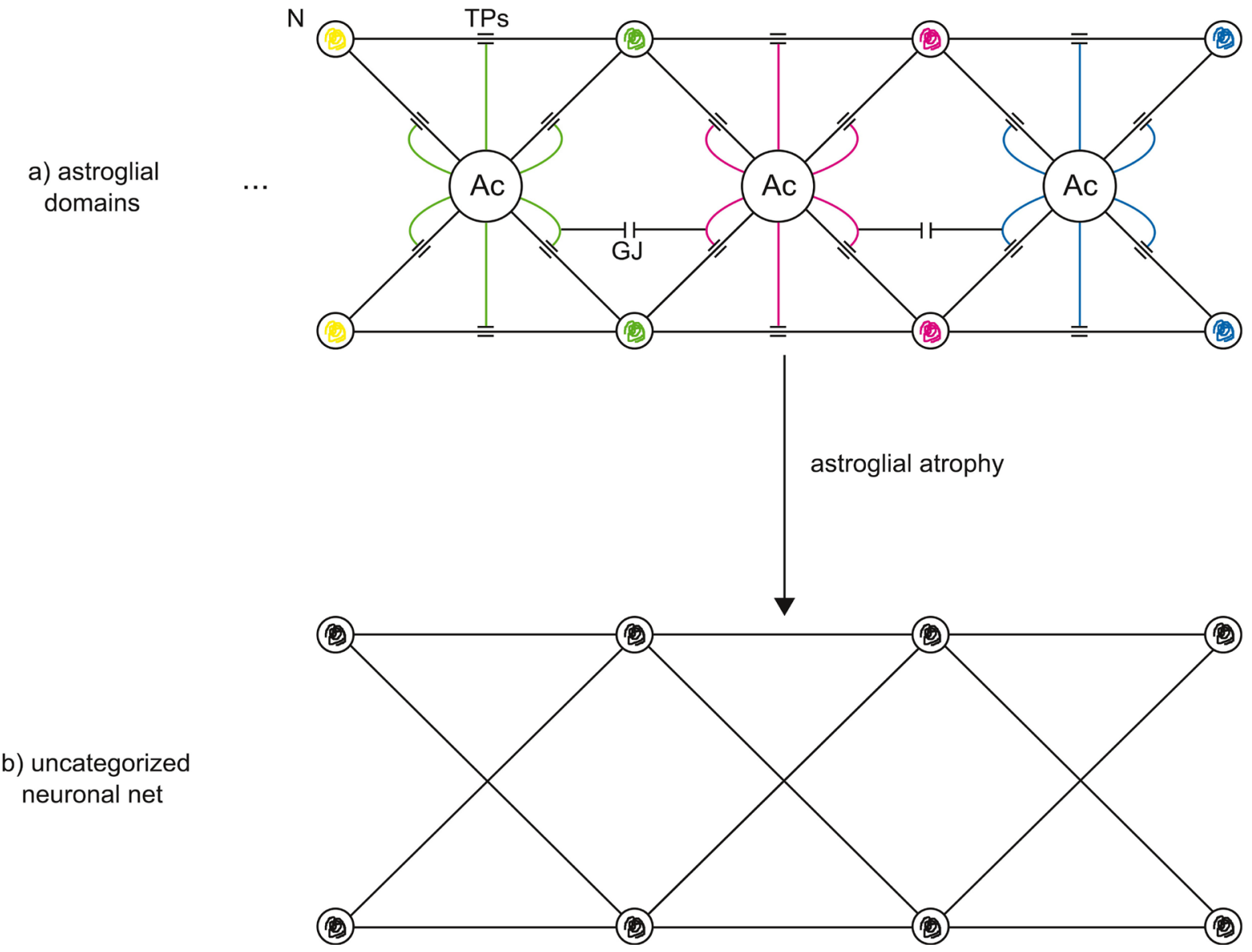

Figure 5. Loss of astroglial domains caused by astroglial atrophy. 
this stage of the illness synaptic imbalances caused by dysregulations of the astroglial modulatory function may be responsible for the onset of the schizophrenic symptomatology. However, if synaptic transmission is totally imbalanced, delusions and hallucinations occur, since synaptic information processing is unmodulated and the information flux becomes unconstrained [17] [18]. Here, the structure of tripartite synapses is not significantly impaired, but astrocyte-synapse interactions become interrupted. Corresponding to my clinical experience tests on patients with schizophrenia demonstrate that they are unable to organize perceptions into categories, e.g. to distinguish facial emotional expression [98]. Note, the loss of the astroglial domain organization is based on the disintegration of domains and not by astrocytosis such as found in epilepsy [99]. However, if the astrodegeneration or atrophy progresses, the boundaries between astroglial domains become loosen up, accompanied by severe cognitive impairment.

Considering again the formal representation of the astroglial domain organization in Figure 3, domains of the same and different qualities are generated in a hierarchical order with increasing complexity. I suggest that the organization of astroglial domains into definite functional and structural units (modules) may enable the brain to comprehend ontological realms in the environment in their distinct quality. This elementary cognitive capability is lost in schizophrenia. Phenomenologically, the ontological confusion of astroglial domain boundaries progressively disorganizes thought processes and social behavior [100]. Together, Figure 6 outlines the components of the model of schizophrenia proposed

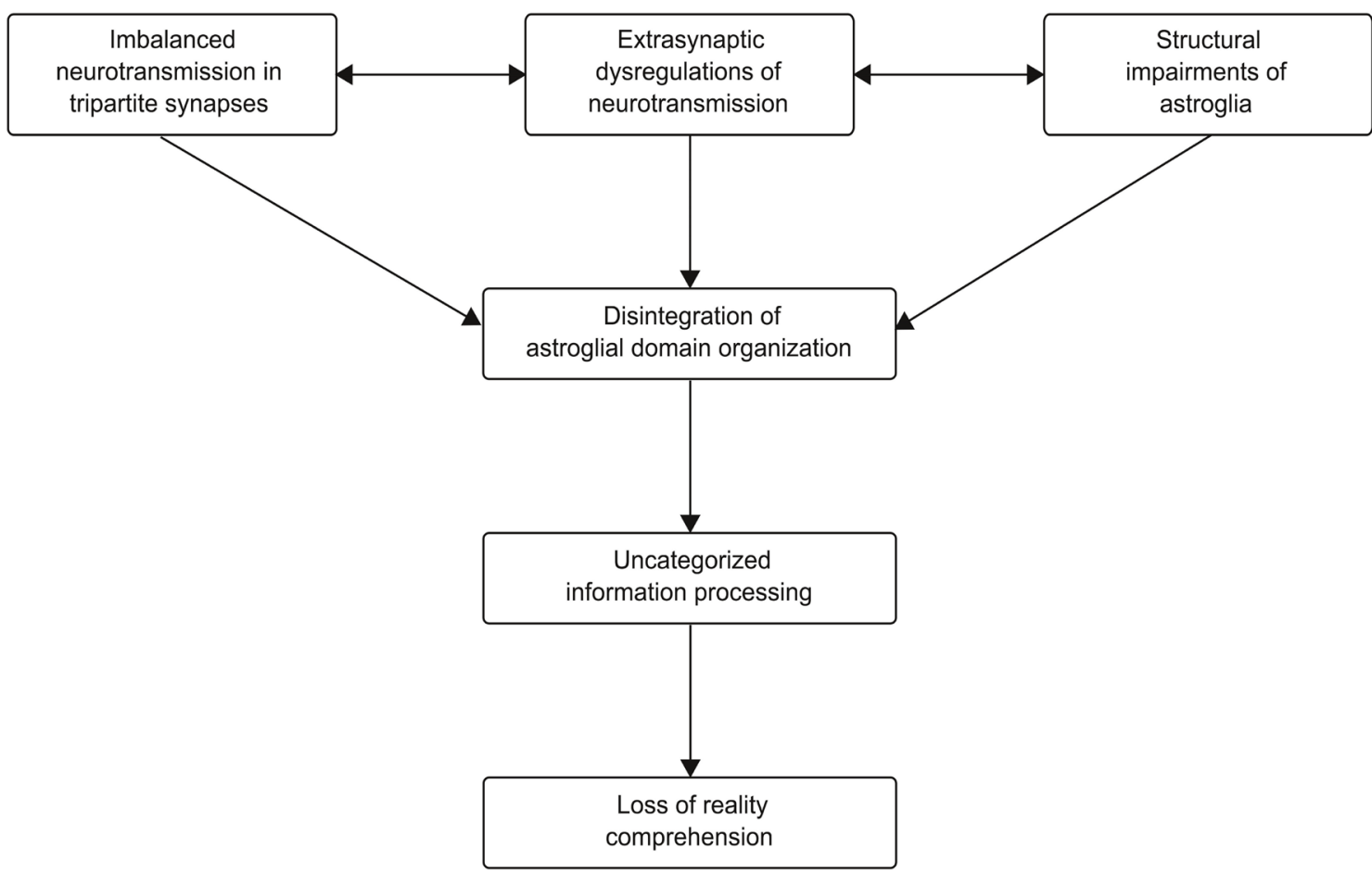

Figure 6. Disintegration of the astroglial domain organization, responsible for the loss of reality comprehension in schizophrenia. 
here. Imbalanced neurotransmission in tripartite synapses, extrasynaptic dysregulation of neurotransmission, and structural impairments of astroglia disintegrate the astroglial domain organization. This leads to uncategorized information processing, responsible for the loss of reality comprehension in schizophrenia.

\section{Conclusions}

The present study attempts to show that the significance of the astroglial domain organization may be based on qualitative information processing by structuring information into categories. This capability of the brain is lost in schizophrenia so that these patients cannot distinguish between Self and Others.

Indeed, experimental findings in the neuronal system indicate that synaptic imbalances and disconnections of the neuronal networks in schizophrenic brains also play a significant role [101] [102]. Importantly, research based on functional magnetic resonance on the neural basis of self-other recognition impairments in schizophrenia provides evidence of dysfunctions in processing of contextual information [103] [104]. Therefore, one might argue that the information categorizing function of astroglia is not necessary, since the neuronal system alone is able to compartmentalize information from reality. However, the modulation and integration of information processing in astroglial domains may represent an extra layer of complexity of computation in the brain [39] [40] [65].

Basically, the "astroglial domain hypothesis of schizophrenia" is testable with optogenetics combined with imaging techniques and chemogenetics in vivo [105], but it is faced with limitations. Whereas astroglial cellular domains have been identified in sections of resected surgical samples of human temporal cortex and also in rodents in vivo, microdomains and their role in information processing cannot presently be identified with brain-imaging methods in vivo. A promising alternative for testing the model proposed here is computer simulation of the astroglial domain organization [106] which may enable to deduce the effects of disintegration of astroglial domains on the pathophysiology of schizophrenia.

Despite experimental limitations the importance of my model may lie in its ability to explain schizophrenic behavior. To the best of my knowledge, this is the first biological model that can elucidate why patients with schizophrenia are unable to distinguish between themselves and others, and why these patients cannot really communicate and without treatment progressively fall into social isolation.

\section{Acknowledgements}

I am very grateful to Birgitta Kofler-Westergren for preparing the final version of the paper and to Christian Streili for designing the figures.

\section{Conflicts of Interest}

The author declares no conflicts of interest. 


\section{References}

[1] Lakhan, S.E. and Vieira, K.F. (2009) Schizophrenia Pathophysiology: Are We Any Closer to a Complete Model? Annals of General Psychiatry, 8, 12. https://doi.org/10.1186/1744-859X-8-12

[2] Jablensky, A. (2010) The Diagnostic Concept of Schizophrenia: Its History, Evolution, and Future Prospects. Dialogues in Clinical Neuroscience, 12, 271-287.

[3] American Psychiatric Association (2013) Diagnostic and Statistical Manual of Mental Disorders. 5th Edition. American Psychiatric Association, Washington DC.

[4] Hales, R.E., Yudofsky, S.C. and Talbott, J.A. (1999) The American Psychiatric Press Textbook of Psychiatry. The American Psychiatric Press, Washington DC.

[5] Owen, M.J., Sawa, A. and Mortensen, P.B. (2016) Schizophrenia. The Lancet, 388, 86-97. https://doi.org/10.1016/S0140-6736(15)01121-6

[6] Karl, T. and Arnold, J.C. (2014) Schizophrenia: A Consequence of Gene-Environment Interactions? Frontiers in Behavioral Neuroscience, 8, 435.

https://doi.org/10.3389/fnbeh.2014.00435

[7] Verkhratsky, A., Rodriguez, J.J. and Steardo, L. (2014) Astrogliopathology: A Central Element of Neuropsychiatric Diseases? Neuroscientist, 20, 576-588. https://doi.org/10.1177/1073858413510208

[8] Haller, C.S., Padmanabhan, J.L., Lizano, P. and Keshavan, M (2014) Recent Advances in Understanding Schizophrenia. F1000 Prime Reports, 6, 57. https://doi.org/10.12703/P6-57

[9] Vadakkan, K.I. (2012) A Structure-Function Mechanism for Schizophrenia. Frontiers in Psychiatry, 3, 108. https://doi.org/10.3389/fpsyt.2012.00108

[10] Bernstein, H.G., Steiner, J., Guest, P.C., Dobrowolny, H. and Bogerts, B. (2015) Glia Cells as Key Players in Schizophrenia Pathology: Recent Insights and Concepts of Therapy. Schizophrenia Research, 161, 4-18. https://doi.org/10.1016/j.schres.2014.03.035

[11] Wang, C., Aleksic, B. and Ozaki, N. (2015) Glia-Related Genes and Their Contribution to Schizophrenia. Psychiatry and Clinical Neurosciences, 69, 448-461. https://doi.org/10.1111/pcn.12290

[12] Verkhratsky, A., Steardo, L., Peng, L. and Parpura, V. (2016) Astroglia, Glutamatergic Transmission and Psychiatric Diseases. In: Schousboe, A. and Sonnewald, U., Eds., The Glutamate/GABA Cycle: Advances in Neurobiology, Springer, Berlin, 307-326. https://doi.org/10.1007/978-3-319-45096-4_12

[13] Mitterauer, B.J. (2003) The Loss of Self-Boundaries: Towards a Neuromolecular Theory of Schizophrenia. BioSystems, 72, 209-215. https://doi.org/10.1016/S0303-2647(03)00144-8

[14] Mitterauer, B. (2005) Nonfunctional Glial Binding Proteins in Tripartite Synapses: A Pathophysiological Model of Schizophrenia. Neuroscientist, 11, 192-198. https://doi.org/10.1177/1073858404265745

[15] Mitterauer, B. (2005) Verlust der Selbst-Grenzen. Entwurf einer interdisziplinären Theorie der Schizophrenie. Springer, Vienna, New York.

[16] Mitterauer, B. (2010) Synaptic Imbalances in Endogenous Psychoses. BioSystems, 100, 113-121. https://doi.org/10.1016/j.biosystems.2010.02.006

[17] Mitterauer, B.J. (2011) Possible Role of Glia in Cognitive Impairment in Schizophrenia. CNS Neuroscience and Therapeutics, 17, 333-334. https://doi.org/10.1111/j.1755-5949.2009.00113.x 
[18] Mitterauer, B.J.(2014) Pathophysiology of Schizophrenia Based on Impaired Glial-Neuronal Interactions. Open Journal of Medical Psychology, 3, 126-140. https://doi.org/10.4236/ojmp.2014.32016

[19] Mitterauer, B.J. and Kofler-Westergren, B. (2011) Possible Effects of Synaptic Imbalances on Oligodendrocyte-Axonic Interactions in Schizophrenia: A Hypothetical Model. Frontiers in Psychiatry, 2, 15. https://doi.org/10.3389/fpsyt.2011.00015

[20] Mitterauer, B. (2007) The Incoherence Hypothesis of Schizophrenia: Based on Decomposed Oligodendrocyte-Axonic Relations. Medical Hypotheses, 69, 1299-1304. https://doi.org/10.1016/j.mehy.2007.03.024

[21] Robertson, J.M. (2013) Astrocyte Domains and the Three-Dimensional and Seamless Expression of Consciousness and Explicit Memories. Medical Hypotheses, 81, 1017-1024. https://doi.org/10.1016/j.mehy.2013.09.021

[22] Oberheim, N.A., Wang, X., Goldman, S. and Nedergaard, M. (2006) Astrocytic Complexity Distinguishes the Human Brain. Trends in Neuroscience, 29, 547-553. https://doi.org/10.1016/j.tins.2006.08.004

[23] Bushong, E.A., Martone, M.E., Jones, Y.Z. and Ellisman, M.H. (2002) Protoplasmic Astrocytes in CA1 Striatum Radiatum Occupy Separate Anatomical Domains. Journal of Neuroscience, 22, 183-192. https://doi.org/10.1523/JNEUROSCI.22-01-00183.2002

[24] Ogata, K. and Kosaka, T. (2002) Structural and Quantitative Analysis of Astrocytes in the Mouse Hippocampus. Neuroscience, 113, 221-233. https://doi.org/10.1016/S0306-4522(02)00041-6

[25] Halassa, M.M., Fellin, T., Takano, H., Dong, J.H. and Haydon, P.G. (2007) Synaptic Islands Defined by the Territory of a Single Astrocyte. Journal of Neuroscience, 27, 6473-6477. https://doi.org/10.1523/JNEUROSCI.1419-07.2007

[26] Araque, A., Parpura, V., Sanzgiri, R.P. and Haydon, P.G. (1999) Tripartite Synapses: Glia, the Unacknowledged Partner. Trends in Neuroscience, 22, 208-215. https://doi.org/10.1016/S0166-2236(98)01349-6

[27] Bernardinelli,Y., Muller, D. and Nikonenko, I. (2014) Astrocyte-Synapse Structural Plasticity. Neural Plasticity, 2014, Article ID: 232105. https://doi.org/10.1155/2014/232105

[28] Grosche, J., Matuyash, V., Möller, T., Verkhratsky, A., et al. (1999) Microdomains for Neuron-Glia Interaction: Parallel Fiber Signaling to Bergmann Glial Cells. Nature Neuroscience, 2, 139-143. https://doi.org/10.1038/5692

[29] Lefebre, C. and Cohen, H. (2005) Handbook of Categorization. Elsevier, New York.

[30] Parpura, V., Heneka, M.T., Montana, V., Oliet, S.H., et al. (2012) Glial Cells in (Patho)Physiology. Journal of Neurochemistry, 121, 4-27. https://doi.org/10.1111/j.1471-4159.2012.07664.x

[31] Heller, J.P. and Rusakov, D.A. (2015) Morphological Plasticity of Astroglia: Understanding Synaptic Microenvironment. Glia, 63, 2133-2151. https://doi.org/10.1002/glia.22821

[32] Oliet, S.H. and Bonfardin, V.D. (2010) Morphological Plasticity of the Rat Supraoptic Nucleus-Cellular Consequences. European Journal of Neuroscience, 32, 1989-1994. https://doi.org/10.1111/j.1460-9568.2010.07514.x

[33] Perez-Alvarez, A., Navarrete, M., Covelo, A., Martin, E.D. and Araque, A. (2014) Structural and Functional Plasticity of Astrocyte Processes and Dendrite Spine Interactions. Journal of Neuroscience, 34, 12738-12744. 
https://doi.org/10.1523/JNEUROSCI.2401-14.2014

[34] Bellesi, M., deVivo, L., Tononi, G. and Cirelli, C. (2015) Effects of Sleep and Wake on Astrocytes: Clues from Molecular and Ultrastructural Studies. BMC Biology, 13, 66. https://doi.org/10.1186/s12915-015-0176-7

[35] Oberheim, N.A., Takano, T., Han, X., He, W., et al. (2009) Uniquely Hominide Features of Adult Human Astrocytes. Journal of Neuroscience, 29, 3276-3287. https://doi.org/10.1523/JNEUROSCI.4707-08.2009

[36] Srinivasan, R., Huang, B.S., Venugopal, S., Johnston, A.D., et al. (2015) $\mathrm{Ca}^{2+}$ Signaling in Astrocytes from $I p 3 r 2^{-/-}$Mice in Brain Slices and during Startle Responses in Vivo. Nature Neuroscience, 18, 708-717. https://doi.org/10.1038/nn.4001

[37] DiCastro, M.A., Chuquet, J., Liaudet, N., Bhaukaurally, K., et al. (2011) Local Ca ${ }^{2+}$ Detection and Modulation of Synaptic Release by Astrocytes. Nature Neuroscience, 14, 1276-1284. https://doi.org/10.1038/nn.2929

[38] Volterra, A., Liaudet, N. and Savtchouk, I. (2014) Astrocyte $\mathrm{Ca}^{2+}$ Signaling: An Unexpected Complexity. Nature Reviews Neuroscience, 15, 327-335. https://doi.org/10.1038/nrn3725

[39] Papouin, T., Dunphy, J., Tolman, M., Foley, C. and Haydon, P.G. (2017) Astrocytic Control of Synaptic Function. Philosophical Transactions of the Royal Society B, 372, Article ID: 20160154. https://doi.org/10.1098/rstb.2016.0154

[40] Araque, A., Carmignoto, G., Haydon, P.G., Oliet, S.H.R., Robitaille, R. and Volterra, A. (2014) Gliotransmitters Travel in Time and Space. Neuron, 81, 728-739. https://doi.org/10.1016/j.neuron.2014.02.007

[41] Werner, G. and Mitterauer, B.J. (2014) The Dynamics of Neuromodulation. In: Plenz, D. and Niebur, E., Eds., Criticality in Neural Systems, Wiley-VCH, Weinheim, 525-537. https://doi.org/10.1002/9783527651009.ch25

[42] Pereira, A.Jr. and Furlan, F.A. (2010) Astrocytes and Human Cognition: Modeling Information Integration and Modulation of Neuronal Activity. Progress in Neurobiology, 92, 405-420. https://doi.org/10.1016/j.pneurobio.2010.07.001

[43] Hirrlinger, J., Hülsmann, S. and Kirchhoff, F. (2004) Astroglial Processes Show Spontaneous Motility at Active Synaptic Terminals in Situ. European Journal of Neuroscience, 20, 2235-2239. https://doi.org/10.1111/j.1460-9568.2004.03689.x

[44] Kozachkov, L. and Michmizos, K.P. (2017) The Causal Role of Astrocytes in Slow-Wave Rhythmogenesis: A Computational Modeling Study.

[45] Grosche, A., Grosche, J., Tackenberg, M., Scheller, D., et al. (2013). Versatile and Simple Approach to Determine Astrocyte Territories in Mouse Neocortex and Hippocampus. PLOS ONE, 8, e69143. https://doi.org/10.1371/journal.pone.0069143

[46] Panatier, A., Valle, J., Haber, M., Murai, K.K., et al. (2011) Astrocytes Are Endogenous Regulators of Basal Transmission At Central Synapses. Cell, 146, 785-798. https://doi.org/10.1016/j.cell.2011.07.022

[47] Shan, D., Yates, S., Roberts, R.C. and McCullumsmith, R.E. (2014) Astroglia and Severe Mental Illness: A Role of Glutamate Microdomains. In: Parpura, V. and Verkhratsky, A., Eds., Pathological Potential of Neuroglia, Springer, New York, 373-395.

[48] Breslin, K., Wade, J.J., Wong-Lin, K.F., Harkin, J., et al. (2018) Potassium and Sodium Microdomains in Thin Astroglial Processes: A Computational Model Study. PLoS Computational Biology, 14, e1006151. https://doi.org/10.1371/journal.pcbi.1006151 
[49] Papouin, T., Dunphy, J.M., Tolman, M., Dineby, K.T. and Haydon, P.G. (2017) Septal Cholinergic Neuromodulation Tunes the Astrocyte-Dependent Gating of Hippocampal NMDA Receptors to Wakefulness. Neuron, 94, 840-854.e7. https://doi.org/10.1016/j.neuron.2017.04.021

[50] Schummers, J., Yu, H. and Sur, M. (2008) Tuned Responses of Astrocytes and Their Influence on Hemodynamic Signals in the Visual Cortex. Science, 320, 1638-1643. https://doi.org/10.1126/science.1156120

[51] Kettenmann, H. and Zorec, R. (2013) Release of Gliotransmitters and Transmitter Receptors in Astrocytes. In: Kettenmann, H. and Ransom, B.R., Eds., Neuroglia, Oxford University Press, New York, 197-214. https://doi.org/10.1093/med/9780199794591.003.0017

[52] Avery, M.C. and Krichmar, J.L. (2017) Neuromodulatory Systems and Their Interactions: A Review of Models, Theories, and Experiments. Frontiers in Neural Circuits, 11, 108. https://doi.org/10.3389/fncir.2017.00108

[53] Snyder, S. (1996) Drugs and the Brain. Scientific American Library, New York.

[54] Redgrave, P. and Gurney, K. (2006) The Short-Latency Dopamine Signal: A Role in Discovering Novel Actions? Nature Reviews Neuroscience, 7, 967-975. https://doi.org/10.1038/nrn2022

[55] Bromberg-Martin, E.S., Matsumoto, M. and Hikosaka, O. (2010) Dopamine in Motivational Control: Rewarding, Aversive, and Alerting. Neuron, 68, 815-834. https://doi.org/10.1016/j.neuron.2010.11.022

[56] Hasselmo, M.O. (1999) Neuromodulation: Acetylcholine and Memory Consolidation. Trends in Cognitive Science, 3, 351-359. https://doi.org/10.1016/S1364-6613(99)01365-0

[57] Yu, A.J. and Dayan, P. (2005) Uncertainty, Neuromodulation, and Attention. Neuron, 46, 681-692. https://doi.org/10.1016/j.neuron.2005.04.026

[58] Picciotto, M.R., Higley, M.J. and Mineur, Y.S. (2012) Acetylcholine as a Neuromodulator: Cholinergic Signaling Shapes Nervous System Function and Behavior. Neuron, 76, 116-129. https://doi.org/10.1016/j.neuron.2012.08.036

[59] Aston-Jones, G. and Cohen, J.D. (2005) An Integrative Theory of Locus Coeruleus-Norepinephrine Function: Adaptive Gain and Optimal Performance. Annual Reviews of Neuroscience, 28, 403-450. https://doi.org/10.1146/annurev.neuro.28.061604.135709

[60] Paukert, M., Argazval, A., Cha, J., Doze, V.A., et al. (2014) Norepinephrine Controls Astroglial Responsiveness to Local Circuit Activity. Neuron, 82, 1263-1270. https://doi.org/10.1016/j.neuron.2014.04.038

[61] O’Donell, J., Zeppenfeld, D., McConnell, E., Pena, S., Nedergaard, M. (2012) Norepinephrine: A Neuromodulator That Boosts the Function of Multiple Cell Types to Optimize CNS Performance. Neurochemical Research, 37, 2496-24512. https://doi.org/10.1007/s11064-012-0818-x

[62] Nadim, F. and Bucher, D. (2014) Neuromodulation of Neurons and Synapses. Current Opinion in Neurobiology, 29, 48-56.

https://doi.org/10.1016/j.conb.2014.05.003

[63] Guenther, G. (1962) Cybernetic Ontology and Transjunctional Operations. In: Yovits, M.C., et al., Eds., Self-Organizing Systems, Spartan Books, Washinghton DC, 313-392.

[64] Mitterauer, B.J. (2013) The Proemial Synapse: Consciousness-Generating Glial-Neuronal Units. In: Pereira, A. and Lehmann, D., Eds., The Unity of Mind, Brain and World, Cambridge University Press, Cambridge, 233-264. 
https://doi.org/10.1017/CBO9781139207065.009

[65] Fellin, T. and Carmignoto, G. (2004) Neuron-to-Astrocyte Signaling in the Brain Represents a Distinct Multifunctional Unit. Journal of Physiology, 559, 3-15. https://doi.org/10.1113/jphysiol.2004.063214

[66] Oliveira, J.F., Sardinka, V.M., Guerra-Jones, S., Araque, A. and Sousa, N. (2015) Do Stars Govern Our Actions? Astrocyte Involvement in Rodent Behavior. Trends in Neuroscience, 38, 535-549. https://doi.org/10.1016/j.tins.2015.07.006

[67] Mitterauer, B.J. (2012) Qualitative Information Processing in Tripartite Synapses: A Hypothetical Model. Cognitive Computation, 4, 181-194. https://doi.org/10.1007/s12559-011-9115-2

[68] Chelini, G., Pantazopoulos, H., Durning, P. and Beretta, S. (2018) The Tetrapartite Synapse: A Key Concept in the Pathophysiology of Schizophrenia. European Psychiatry, 60, 60-63. https://doi.org/10.1016/j.eurpsy.2018.02.003

[69] Windrem, M.S., Osipovitch, M., Liu, Z., Bates, J., et al. (2017) Human iPSC Glial Mouse Chimeras Reveal Glial Contribution to Schizophrenia. Cell Stem Cell, 21, 195-208. https://doi.org/10.1016/j.stem.2017.06.012

[70] Covelo, A, and Araque, A. (2018) Neuronal Activity Determines Distinct Gliotransmitter Release from a Single Astrocyte. Elife Sciences, 7, e32237. https://doi.org/10.7554/eLife.32237

[71] Hashimoto, K., Fukushima, T., Shimizu, E., Komatsu, N., et al. (2003) Decreased Serum Levels of D-Serine in Patients with Schizophrenia: Evidence in Support of the $N$-Methyl-D-Asparate Receptor Hypofunction Hypothesis of Schizophrenia. Archives of General Psychiatry, 60, 572-576. https://doi.org/10.1001/archpsyc.60.6.572

[72] Bendikov, I., Nadri, C, Amar, S., Panizutti, R., et al. (2007) A CSF and Postmortem Brain Study of D-Serine Metabolic Parameters in Schizophrenia. Schizophrenia Research, 90, 41-51. https://doi.org/10.1016/j.schres.2006.10.010

[73] Ma, T.M., Abazyan, B., Nomura, J., et al. (2013) Pathogenic Disruption of DISC1-Serine Racemase Binding Elicits Schizophrenia-Like Behaviors via D-Serine Depletion. Molecular Psychiatry, 18, 557-567. https://doi.org/10.1038/mp.2012.97

[74] Xia, M., Abazyan, S., Jouroukhin, Y. and Pletnikov, M. (2016) Behavioral Sequelae of Astrocyte Dysfunction: Focus on Animal Models of Schizophrenia. Schizophrenia Research, 176, 72-82. https://doi.org/10.1016/j.schres.2014.10.044

[75] Pantazopoulos, H., Woo, T.U., Lim, M.P., Lange, N. and Beretta, S. (2010) Extracellular Matrix-Glial Abnormalities in the Amygdala and Entorhinal Cortex of Subjects Diagnosed with Schizophrenia. Archives of General Psychiatry, 67, 155-166. https://doi.org/10.1001/archgenpsychiatry.2009.196

[76] Schwarcz, R. and Hunter, C.A. (2007) Toxoplasma Gondii and Schizophrenia: Linkage through Astrocyte-Derived Kynurenic Acid? Schizophrenia Bulletin, 33, 652-653.

[77] Plitman, E., Iwata, Y., Carovaggio, F., Nakajima, S., et al. (2017) Kynurenic Acid in Schizophrenia: A Systematic Review and Meta-Analysis. Schizophrenia Bulletin, 43, 764-777. https://doi.org/10.1093/schbul/sbw221

[78] Labrie, V., Wong, A.H. and Roder, J.C. (2012) Contributions of the D-Serine Pathway to Schizophrenia. Neuropharmacology, 62, 1484-1503.

https://doi.org/10.1016/j.neuropharm.2011.01.030

[79] Ripke, S., Neale, B., Corvin, A., Walters, J., et al. (2014) Schizophrenia Working Group of the Psychiatric Genomics Consortium. Biological insights from 108 Schi- 
zophrenia-Associated Genetic Loci. Nature, 511, 421-427.

https://doi.org/10.1038/nature13595

[80] Yang, Y., Paspalas, C.D., Jin, L.E., Piccioto, M.R., et al. (2013) Nicotinic a7 Receptors Enhance NMDA Cognitive Circuits in Dorsolateral Prefrontal Cortex. Proceedings of the National Academy of Sciences of the United States of America, 110, 12078-12083. https://doi.org/10.1073/pnas.1307849110

[81] Zappettini, S., Grilli, M., Olivero, G., Chen, J., et al. (2014) Nicotinic a7 Receptor Activation Subjectively Potentiates the Function of NMDA Receptors in Glutamatergic Terminals of the Nucleus Accumbens. Frontiers in Cellular Neuroscience, 8 , 332. https://doi.org/10.3389/fncel.2014.00332

[82] Mahdavi, A., Bahrami, F. and Janahmadi, M. (2015) Analysis of Impaired LTP in Schizophrenia Using an Extended Mathematical Model of a Tripartite Synapse. 22nd Iranian Conference on Biomedical Engineering (ICBME), Tehran, 25-27 November 2015, 76-80. https://doi.org/10.1109/ICBME.2015.7404120

[83] Ohnuma, T., Tessler, S., Avai, H., Faull, R.L., et al. (2000) Gene Expression of Metabotropic Glutamate Receptor 5 and Excitatory Amino Acid Transporter 2 in the Schizophrenic Hippocampus. Molecular Brain Research, 85, 24-31. https://doi.org/10.1016/S0169-328X(00)00222-9

[84] Bauer, D., Haroutunian, V., Meador-Woodruff, J.H. and McCullumsmith, R.E. (2010) Abnormal Glycosylation of EAAT1 and EAAT2 in Prefrontal Cortex of Elderly Patients with Schizophrenia. Schizophrenia Research, 117, 92-98. https://doi.org/10.1016/j.schres.2009.07.025

[85] Rao, J.S., Kellom, M., Reese, E.A., Rapoport, S.I. and Kim, H.W. (2012) Dysregulated Glutamate and Dopamine Transporters in Postmortem Frontal Cortex from Bipolar and Schizophrenic Patients. Journal of Affective Disorders, 136, 63-71. https://doi.org/10.1016/j.jad.2011.08.017

[86] Hu, W., McDonald, M.L., Elswick, D.E. and Smeet, R.A. (2015) Cell-Specific Abnormalities of Glutamate Transporters in Schizophrenia. Annals of the New York Academy of Science, 1338, 38-57. https://doi.org/10.1111/nyas.12547

[87] Karlsson, R.M., Tanaka, K., Saksida, L.M., Bussey, T.J., et al. (2009) Assessment of Glutamate Transporter GLAST (EAAT1)-Deficient Mice for Phenotypes Relevant to the Negative and Executive Cognitive Symptoms of Schizophrenia. Neuropsychopharmacology, 34, 1578-1589. https://doi.org/10.1038/npp.2008.215

[88] Hashimoto, K., Engberg, G., Shimizu, E., Nordin, C. and Lindstrom, L.H. (2005) Elevated Glutamate/Glutamine Ratio in Cerebrospinal Fluid of First Episode and Drug Naive Schizophrenic Patients. BMC Psychiatry, 5, 6. https://doi.org/10.1186/1471-244X-5-6

[89] Baker, D.A., Madayag, A., Kristiansen, L.V., Meador-Woodruff, J.H., et al. (2008) Contribution of Cystine-Glutamate Antiporters to the Psychotomimetic Effects of Phencyclidine. Neuropsychopharmacology, 33, 1760-1772.

https://doi.org/10.1038/sj.npp.1301532

[90] Rajkowska, G., Miguel-Hidalgo, J.J., Makkos, Z., Meltzer, H., et al. (2002) Layer-Specific Reduction of GFAP-Reactive Astroglia in the Dorsolateral Prefrontal Cortex in Schizophrenia. Schizophrenia Research, 57, 127-138.

https://doi.org/10.1016/S0920-9964(02)00339-0

[91] Williams, M.R., Hampton, T., Pearce, R.K., Hirsch, S.R., et al. (2013) Astrocyte Decrease in the Subgenual Cingulate and Callosal Genu in Schizophrenia. European Archives of Psychiatry and Clinical Neuroscience, 263, 41-52. https://doi.org/10.1007/s00406-012-0328-5 
[92] Kolomeets, N.S. and Uranova, N. (2010) Ultrastructural Abnormalities of Astrocytes in the Hippocampus in Schizophrenia and Duration of Illness: A Postmortem Morphometric Study. World Journal of Biological Psychiatry, 11, 282-292. https://doi.org/10.3109/15622970902806124

[93] Takahashi, N. and Sakurai, T. (2013) Roles of Glial Cells in Schizophrenia: Possible Targets for Therapeutic Approaches. Neurobiological Disorders, 53, 49-60. https://doi.org/10.1016/j.nbd.2012.11.001

[94] Spangaro, M., Bosia, M., Zanoletti, A., Bechi, M., et al. (2012) Cognitive Dysfunction and Glutamate Uptake: Effect of EAAT2 Polymorphism in Schizophrenia. Neuroscience Letters, 522, 151-155. https://doi.org/10.1016/j.neulet.2012.06.030

[95] Berretta, S. (2012) Extracellular Matrix Abnormalities in Schizophrenia. Neuropharmacology, 62, 1584-1597.

https://doi.org/10.1016/j.neuropharm.2011.08.010

[96] Südhof, T.C. (2008) Neuroligins and Neurexins Link Synaptic Function to Cognitive Disease. Nature, 455, 903-911. https://doi.org/10.1038/nature07456

[97] George, M., Maheshwari, S., Chandran, S., Monokar, J.S. and Rao, T.S. (2017) Understanding the Schizophrenia Prodrome. Indian Journal of Psychiatry, 59, 505-509.

[98] Holden, C. (2003) Deconstructing Schizophrenia. Science, 299, 333-335. https://doi.org/10.1126/science.299.5605.333

[99] Oberheim, N.A., Tian, G.F., Han, X., Peng, W., et al. (2008) Loss of Astrocytic Domain Organization in the Epileptic Brain. Journal of Neuroscience, 28, 3264-3276. https://doi.org/10.1523/JNEUROSCI.4980-07.2008

[100] Ebish, S.H., Salone, A., Ferri, F., DeBerardis, D., et al. (2013) Out of Touch with Reality? Social Perception in First-Episode Schizophrenia. Social Cognitive and Affective Neuroscience, 8, 394-403. https://doi.org/10.1093/scan/nss012

[101] Friston, K., Brown, H.R., Liemerkus, J. and Stephan, K.E. (2016) The Disconnection Hypothesis. Schizophrenia Research, 176, 83-94. https://doi.org/10.1016/j.schres.2016.07.014

[102] Goghari, V.M., Sponheim, S.R. and MacDonald, A.W. (2010) The Functional Neuroanatomy of Symptom Dimensions in Schizophrenia: A Qualitative and Quantitative Review of a Persistent Question. Neuroscience Biobehavioral Reviews, 34, 468. https://doi.org/10.1016/j.neubiorev.2009.09.004

[103] Lee, J.S., Chun, J.W., Lee, S.H., Kim, E., et al. (2015) Altered Neural Basis of the Reality Processing and Its Relation to Cognitive Insight in Schizophrenia. PLoS ONE, 10, e0120478. https://doi.org/10.1371/journal.pone.0120478

[104] Keromnes, G., Motillon, T., Coulon, N., Berthoz, A., et al. (2018) Self-Other Recognition Impairments in Individuals with Schizophrenia: A New Experimental Paradigm Using Double Mirror. NPJ Schizophrenia, 4, 24. https://doi.org/10.1038/s41537-018-0065-5

[105] Losi, G., Mariotti, L., Sessolo, M. and Carmignoto, G. (2017) New Tools to Study Astrocyte $\mathrm{Ca}^{2+}$ Signal Dynamics in Brain Networks in Vivo. Frontiers in Cellular Neuroscience, 11, 134. https://doi.org/10.3389/fncel.2017.00134

[106] DePitta, M., Volman, V., Berry, H., Parpura, K., et al. (2012) Computational Quest for Understanding the Role of Astrocyte Signaling in Synaptic Transmission and Plasticity. Frontiers in Computational Neuroscience, 6, 98. 